\title{
Blood Feeding Preference of Female Aedes aegypti Mosquitoes for Human Blood Group Types and Its Impact on Their Fecundity: Implications for Vector Control
}

\author{
Manushika Prasadini ${ }^{1}$, Dilakshini Dayananda ${ }^{1}$, Sachini Fernando ${ }^{1}$, Iresha Harischandra ${ }^{1,2}$, \\ Nissanka De Silva ${ }^{1, *}$ \\ ${ }^{1}$ Centre for Biotechnology, Department of Zoology, Faculty of Applied Sciences, University of Sri Jayewardenepura, Gangodawila, Sri \\ Lanka \\ ${ }^{2}$ Genetics and Molecular Biology Unit, Faculty of Applied Sciences, University of Sri Jayewardenepura, Gangodawila, Sri Lanka
}

\author{
Email address: \\ manushikaprasadini@gmail.com (M. Prasadini), dilakshini@sci.sjp.ac.lk (D. Dayananda), sachini@sci.sjp.ac.lk (S. Fernando), \\ iresha@sci.sjp.ac.lk (I. Harischandra),nissanka@sci.sjp.ac.lk (N. De Silva) \\ ${ }^{*}$ Corresponding author
}

\section{To cite this article:}

Manushika Prasadini, Dilakshini Dayananda, Sachini Fernando, Iresha Harischandra, Nissanka De Silva. Blood Feeding Preference of Female Aedes aegypti Mosquitoes for Human Blood Group Types and Its Impact on Their Fecundity: Implications for Vector Control. American Journal of Entomology. Vol. 3, No. 2, 2019, pp. 43-48. doi: 10.11648/j.aje.20190302.13

Received: May 18, 2019; Accepted: June 13, 2019; Published: June 26, 2019

\begin{abstract}
Aedes aegypti is the major vector of Dengue in Sri Lanka. Dengue virus is transmitted via blood feeding of vector mosquitoes. Blood is an essential component for the fecundity of mosquitoes and the knowledge on feeding behaviour of $A e$. aegypti is important in dengue prevention measures. Therefore, the study was designed to identify the preferential feeding of Ae. aegypti on human $\mathrm{ABO}$ blood groups and their impacts on fecundity. Laboratory reared female Ae. aegypti mosquitoes were exposed to all four blood groups at once in separate membrane feeders. After feeding, DNA of blood in mosquitoes was extracted and identified using ABO genotyping PCR. Fecundity was determined by the mean number of eggs in an egg batch. The highest preference was observed for the blood group " $\mathrm{O}$ " $(\mathrm{P}<0.05 ; 0.00)$. The mean number of egg production of each blood group was not significantly different showing no impact on the blood type for the fecundity. This study reports for the first time that there is no direct impact of the preferred blood group on the fecundity of Ae. aegypti female mosquitoes. Furthermore, preference of blood group selection by female mosquitoes would be highly important in personal protection measures against mosquito bites, as the personal protection is one of the most effective control measures for prevention of Dengue.
\end{abstract}

Keywords: Aedes aegypti, Dengue, ABO Blood Groups, Feeding Behaviour, Fecundity

\section{Introduction}

Aedes aegypti, major vector of Dengue, Yellow fever and Chikungunaya is currently distributed throughout the tropics including Sri Lanka and a number of subtropical regions such as South-Eastern United States, the Middle East, Southeast Asia, the Pacific and Indian Islands and Northern Australia [1]. It is considered as the major vector of epidemic dengue in Sri Lanka causing around 185, 969 cases and 215 deaths in 2017 [2]. The severity and prevalence of the dengue disease is increasing and epidemics are reporting continuously in Sri
Lanka. Since a specific drug to treat is unavailable and the potential vaccine candidates are still under developmental stages, vector control personal protection against mosquito bites is the only available effective method to control the severity of epidemic dengue.

Dengue virus is transmitted by the bites of infectious $A e$. aegypti mosquitoes. After mating, female Ae. aegypti needs to feed on a vertebrate blood meal as the source of nutrients for their fecundity [3]. Hence, selection of a good blood source is essential for their reproductive success. Therefore, blood feeding patterns of the mosquitoes provide important information on disease transmission via biting of mosquitoes 
and could be useful in effective vector control strategies.

Detection of host species by blood meal analysis of different wild caught Ae. aegypti and Ae. albopictus (potential secondary vector of dengue in Sri Lanka) has been carried out by using various methods $[4,5,6,7]$. Ae. aegypti has reported preferentially selecting human blood than other vertebrate hosts as their post mating blood meal [8, 9]. Among the preference for human $\mathrm{ABO}$ blood groups, Anopheles gambiae has shown attraction to $\mathrm{AB}$ group [10] and the Ae. albopictus to $\mathrm{O}$ group [11]. However, the influence on blood type for mosquito fecundity has not been reported.

Personal protection to prevent mosquito bites is one of the key factors to the control the dengue via preventing the connection between the dengue virus and the human host [12]. This is crucial because the silent transmission via asymptomatic carriers plays a major role in dengue spread [13]. Knowledge on selection of blood group and the effect on fecundity is essential factors to be used in effective personal protecting vector control methods under the national dengue prevention and control planning in Sri Lanka. Therefore, the current study has designed to determine the most selective human blood type by dengue vector $A e$. aegypti mosquitoes and the influence of the blood type for their fecundity.

\section{Methodology}

\subsection{Mosquitoes}

Aedes aegypti mosquito colony was established in the Department of Zoology, University of Sri Jayewardenepura, Sri Lanka, from a single engorged wild mosquito collected from Maharagama (N 6.854800, E 79.928604) area. The mosquitoes in the colony were maintained under a $12: 12$ (light: dark) cycle, $28^{\circ} \mathrm{C}$ constant temperature and $80 \%$ relative humidity. Mosquitoes were reared using standard conditions [14] to generate similar size individuals. Adult mosquitoes were housed in $15 \times 15 \times 30 \mathrm{~cm}$ mosquito cages with mesh screening on top, provided with a $20 \%$ sugar solution and water ad libitum. The eggs laid by the female mosquitoes of $12^{\text {th }}$ generation of the colony were used in the current study.

\subsubsection{Mosquito Rearing and Maintenance}

The mosquito rearing was carried out in the insectary. Water was boiled for deoxygenating and poured into $250 \mathrm{ml}$ volume glass bottles. Then the bottles were allowed to become room temperature with loosely closed lids. The eggs of female Ae. aegypti obtained from the colony were submerged in deoxygenated water to induce their hatching [15]. One day old larvae were transferred to the water filled enamel trays. Larvae were fed with commercially available fish feed at the rate of $0.32 \mathrm{mg}$ per larva until $4^{\text {th }}$ instar stage. Trays were daily inspected for pupae and they were separated into plastic cups and transferred into the adult cages for emerging adults.

After the emergence of adults, 100 mosquitoes each (50 males: 50 females) were transferred to six (6) new cages (15 x $15 \times 30 \mathrm{~cm}$ ) top covered with a net, using a mouth aspirator. Constant 1:1 sex ratio was maintained in every cage to ensure the mating of all females [16]. Sucrose solution $(10 \%)$ soaked cotton pads were provided on the top of the cage as the food source for adults [17]. In the insectary, $28^{\circ} \mathrm{C}$ temperature and $80 \%$ relative humidity was maintained continuously.

\subsubsection{Blood Feeding of Mosquitoes}

Five days old 50 female mosquitoes were separated into a new cage $(20 \times 20 \times 20 \mathrm{~cm})$ with a mesh on the top and starved for $16 \mathrm{~h}$ without providing any type of food source or water [15]. Feeding with blood sources was carried out via artificial membrane feeder. The membrane feeding system was composed with water jacketed glass feeders and a water circulating system. Commercially available murine monoclonal anti sera method was used to determine the group of the blood samples. The bottoms of four glass feeders were covered by parafilm (Marrifield, USA) membranes. The circulating water in the system was maintained at constant $37^{\circ} \mathrm{C}$ temperature. Four glass feeders with $3 \mathrm{ml}$ of human blood in types $\mathrm{A}, \mathrm{B}, \mathrm{AB}$ and $\mathrm{O}$ were used for feeding. The feeders with blood was connected to the water circulating system and kept 30 minutes to become equal temperature of blood and circulating water. Mosquitoes were allowed to feed for one hour. After the feeding process, engorged mosquitoes were chilled immediately and keep frozen until extract DNA. This feeding was carried out in six (6) cages separately to ensure the feeding of all 300 female mosquitoes which allowed for mating.

\subsection{Extraction of DNA from Human Blood Ingested by the Mosquitoes}

The frozen mosquitoes were placed in room temperature about 30 minutes for thawing. The mosquito abdomens were separated. Samples were homogenized in $100 \mu$ l of extraction buffer [9]. After one hour incubation at $65^{\circ} \mathrm{C}$, samples were treated with $8 \mathrm{M}$ cold potassium acetate and incubated on ice for 45 minutes. DNA was precipitated using 100\% ethyl alcohol. DNA was stored at $-20{ }^{\circ} \mathrm{C}$ until further use. Same procedure was carried out to extract DNA from four types of blood groups to use as positive controls to ensure the accurate amplification for each blood type.

The relative quality of the all extracted DNA was evaluated by detecting the presence of human DNA in a PCR amplification using human specific primers, Human 741F, UNREV 1025 [7]. A 334 bp PCR fragment was expected for the detection of human blood. PCR mixture was consisted of 1X PCR buffer, $1.25 \mathrm{U}$ of Taq Polymerase, $2.5 \mathrm{mM} \mathrm{MgCl}_{2}$, $0.8 \mathrm{mM}$ dNTP, 50 pmol of primers and 10ng of template DNA in $25 \mu \mathrm{l}$. Cyclic conditions were $5 \mathrm{~min}$ at $95^{\circ} \mathrm{C}, 35$ cycles of $1 \mathrm{~min}$ at $95^{\circ} \mathrm{C}, 1 \mathrm{~min}$ at $58^{\circ} \mathrm{C}$ and $1 \mathrm{~min}$ at $72^{\circ} \mathrm{C}$ with a final extension of $7 \mathrm{~min}$ at $72^{\circ} \mathrm{C}$.

\subsection{ABO Genotyping of Mosquito Blood Meal}

Each sample of DNA isolated from blood in mosquito gut was amplified for four consecutive PCR reactions to 
determine the blood group using primers designed by Lee et al. [18]. These four different primer combinations (Table 1) were involved in detection of six alleles of the ABO gene; A101, A102, B101, O01, O02 and cis-AB (18]. Each PCR reaction was contained $1 \mathrm{X}$ PCR buffer, $1.25 \mathrm{U}$ of $\mathrm{Taq}$
Polymerase, $4.5 \mathrm{mM} \mathrm{MgCl} 2,0.2 \mathrm{mM}$ dNTP, $0.5 \mu \mathrm{M}$ of each allele specific primer and 10ng of template DNA in $25 \mu 1$. Cyclic conditions were $5 \mathrm{~min}$ at $95^{\circ} \mathrm{C}, 35$ cycles of $40 \mathrm{~s}$ at $95^{\circ} \mathrm{C}, 40 \mathrm{~s}$ at $60^{\circ} \mathrm{C}$ and $40 \mathrm{~s}$ at $72^{\circ} \mathrm{C}$ with a final extension of 5 $\min$ at $72^{\circ} \mathrm{C}$.

Table 1. Primers used in the ABO genotyping.

\begin{tabular}{|c|c|c|c|}
\hline PCR reaction & Primer pair & Expected band size & Allele specificity \\
\hline \multirow{2}{*}{1} & $\begin{array}{l}\text { 261G:5'GCAGTAGGAAGGATGTCCTCGTGtTG3' int6: } \\
\text { 5'-AGACCTCAATGTCCACAGTCACTCG-3' }\end{array}$ & 205 & A101, A102, B101, cis-AB01 \\
\hline & $\begin{array}{l}\text { 467C:5'-CCACTACTATGTCTTCACCGACCAtCC-3' 803G: } \\
\text { 5'-CACCGACCCCCCGAAGAtCC-3' }\end{array}$ & 381 & $A 101,001,002$ \\
\hline \multirow{2}{*}{2} & $\begin{array}{l}\text { 297A:5'-CCATTGTCTGGGAGGGCcCA-3' int6: } \\
\text { 5'-AGACCTCAATGTCCACAGTCACTCG-3' }\end{array}$ & 164 & $A 101, A 102, O 01$, cis- $A B 01$ \\
\hline & $\begin{array}{l}\text { 467C:5'-CCACTACTATGTCTTCACCGACCAtCC-3' 803C: } \\
\text { 5'-CACCGACCCCCCGAAGAtCG-3' }\end{array}$ & 381 & B101 \\
\hline \multirow{2}{*}{3} & $\begin{array}{l}\text { 261A:5'-GCAGTAGGAAGGATGTCCTCGTGtTA-3' int6: } \\
\text { 5'-AGACCTCAATGTCCACAGTCACTCG-3' }\end{array}$ & 205 & 001,002 \\
\hline & $\begin{array}{l}\text { 467T:5'-CCACTACTATGTCTTCACCGACCAtCT-3' 803G: } \\
\text { 5'-CACCGACCCCCCGAAGAtCC-3' }\end{array}$ & 381 & A102 \\
\hline \multirow{2}{*}{4} & $\begin{array}{l}\text { 297G:5'-CCATTGTCTGGGAGGGCcCG-3' int6: } \\
\text { 5'-AGACCTCAATGTCCACAGTCACTCG-3' }\end{array}$ & 164 & B102, O02 \\
\hline & $\begin{array}{l}\text { 467T:5'-CCACTACTATGTCTTCACCGACCAtCT-3' 803C: } \\
\text { 5'-CACCGACCCCCCGAAGAtCG-3' }\end{array}$ & 381 & Cis-AB01 \\
\hline
\end{tabular}

\subsection{Fecundity of Aedes aegypti Engorged Females with Four Blood Groups}

Male and female mosquitoes of generation $\mathrm{F}_{32}$ were used in the experiment. Newly emerged adult mosquitoes $(n=400)$ were transferred into four adult cages $(15 \times 15 \times 30 \mathrm{~cm})$. Each cage was housed with 100 mosquitoes (50:50 male: female). They were provided $20 \%$ sucrose soaked cotton pads as the food source and allowed to mate for five days. After five days, females were separated into new adult cages as 50 females per cage. Then the female mosquitoes of all four cages were starved for $24 \mathrm{~h}$ without providing any food or water. Circulating water system of artificial membrane feeder was connected to four glass feeders (one feeder for one cage) and filled with four different types of blood. Mosquitoes were allowed feeding for one hour.

Fully engorged females were separated into egg laying cages individually (4 cm diameter x $10 \mathrm{~cm}$ height). Cotton pad with $20 \%$ sucrose was provided as the food source. After 7 days, females were removed and eggs were counted using tally counter and hand lens. The entire procedure was replicated for four times. Statistical comparison of the data was carried out using One-way analysis of variance (ANOVA) in Minitab 14 for windows.

\section{Results}

\subsection{Blood Feeding Preference of Aedes aegypti}

Among 300 mosquitoes allowed for feeding, 192 individual females were identified as blood fed mosquitoes by amplifying the $334 \mathrm{bp}$ fragment in the human DNA detection PCR.

In the ABO genotyping PCR, blood groups $A(n=34), B$ $(n=37), \quad A B \quad(n=48)$ and $O \quad(n=59)$ were detected.
Additionally, $7.30 \%$ samples $(n=14)$ were detected having consumed multiple blood meals (Table 2). These samples have amplified respective PCR bands for more than single blood type. The most selected blood group was $\mathrm{O}(30.78 \%)$.

Table 2. Number of blood fed mosquitoes detected from the PCR analysis.

\begin{tabular}{lll}
\hline Blood group & $\begin{array}{l}\text { Number of blood fed } \\
\text { mosquitoes }\end{array}$ & $\begin{array}{l}\text { Mean percentage (\%) } \\
\pm \text { Standard deviation }\end{array}$ \\
\hline A & 34 & $17.60 \pm 2.42$ \\
B & 37 & $19.35 \pm 3.82$ \\
AB & 48 & $24.97 \pm 2.17$ \\
O & 59 & $30.78 \pm 2.00$ \\
Multiple meals & 14 & $7.30 \pm 3.72$ \\
Total & 192 & \\
\hline
\end{tabular}

One way ANOVA to determine the significance of preference among the four blood groups shows significant difference among all four blood groups $(\mathrm{P}<0.05 ; 0.00)$. The preference for the selection of blood meals of Ae. aegypti detected as $\mathrm{O}>\mathrm{AB}>\mathrm{B}>\mathrm{A}$ order (Figure 1).

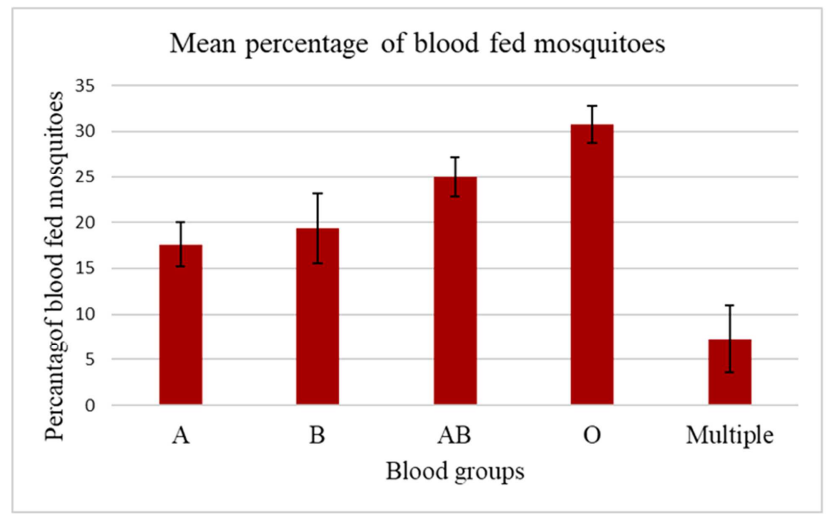

Figure 1. Percentage of Aedes aegypti mosquitoes feeding on the different blood groups. 


\subsection{Effect of Blood Groups on Fecundity of Aedes Aegypti}

The mean numbers of eggs laid per females were analyzed using one way ANOVA test with the 0.05 significance level.
There were no significant difference in fecundity for different blood groups $(\mathrm{P}>0.05 ; 0.937)$. The mean number of eggs laid by different blood group consumers is shown in Table 3 .

Table 3. Aedes aegypti egg production in response to the different blood groups.

\begin{tabular}{llll}
\hline Blood type & Blood fed females out of 200 total mosquitoes & No. of females -Egg laid & Mean no. of eggs/female ( \pm SD) \\
\hline A & 153 & 150 & $46.063 \pm 7.827$ \\
B & 143 & 138 & $43.183 \pm 6.358$ \\
AB & 148 & 142 & $44.463 \pm 4.037$ \\
O & 160 & 155 & $45.225 \pm 7.639$ \\
\hline
\end{tabular}

When allowed ad libitum feeding of separate blood groups for one hour, the highest number of blood fed mosquitoes were reported for O blood group ( $\mathrm{n}=160)$ while 155 of them were laid eggs. Percentage of blood feeding success was between $71.5 \%-80.0 \%$ among four blood groups and there was no significant difference among blood groups $(\mathrm{P}>0.05)$. Similarly the percentage oviposition success was $(69.0 \%$ $77.5 \%)$ not significant $(\mathrm{P}>0.05)$ among four blood groups (Figure 2).

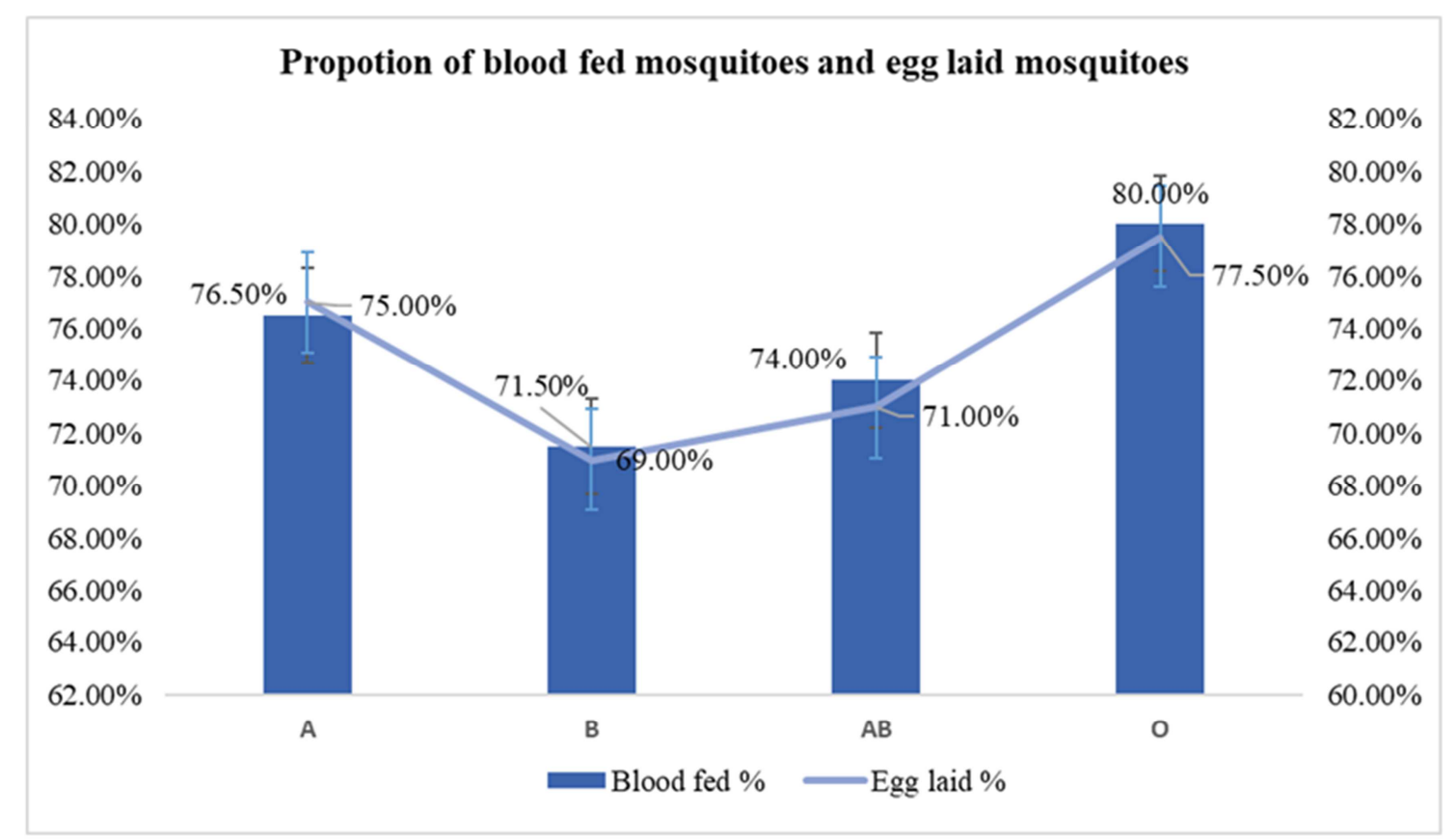

Figure 2. Percentages of blood feeding mosquitoes and subsequent egg laid mosquitoes for each blood group.

\section{Discussion}

The study revealed a high blood feeding preference for blood group type $\mathrm{O}$ in laboratory reared Ae. aegypti mosquitoes in artificial membrane feeding. However, the study confirms the preference for the blood type is not influence for the fecundity of Ae. aegypti female mosquitoes. Similarly, blood group $\mathrm{O}$ is selecting by number of mosquito species; Anopheles gambiae [10] in terms of blood feeding and $A e$. albopictus [11] in terms of landing preference have been reported previously. Ae. aegypti has shown selecting human hosts with blood type $\mathrm{O}$ when provided natural feeding opportunity via human skin [19].

Nearly 1/3 (108 out of 300 individuals) of total female mosquitoes allowed for mating were not blood fed, according to the amplified PCR fragments in the initial human DNA detecting PCR (blood feeding success rate was 64.0\%). However, they were provided all four blood sources at once ad libitum during the one hour feeding time. The fecundity experiment reveals no significant difference in blood feeding success and subsequent oviposition success among four blood groups, when they were provided different types of blood sources separately. This shows that when they have only one choice, feeding was higher than multiple choices. This could be due to the requirement of more time duration for selection over variety of sources to acquire the sufficient amount of most suitable blood meal for their reproductive success. This provides information to assume that the blood meal selection varies with the availability of the number of sources and the time. Similarly Culex species has shown variable selection of blood sources on the availability and the mosquito density [20]. Current study maintained equal mosquito density throughout the study period.

Even though this mosquito prefers $\mathrm{O}$ blood type, no influence showed on fecundity. Selection and blood feeding on human blood may be basically depends on the availability, 
easy access and easy to feed. Moreover, results showed that although the blood group type $\mathrm{O}$ was more favoured than others, considerable ingestion of other blood groups were also observed. The von Willerbrand factors (VWFs) are glycoproteins that are involved in blood haemostasis. The high VWF amounts are responsible to high thrombotic activities which result in blood coagulation [21]. ABO blood groups contain different VWF levels in the blood plasma. Blood group type $\mathrm{O}$ individuals are known to possess $20 \%$ to $30 \%$ lower VWF levels compared to non-blood group type O individuals [22]. Similarly blood group type O - VWF molecules are known to have a short survivorship and are known to clear more rapidly than other molecules belong to other three groups [23]. Supporting this, recent studies have reported over presentation of arterial thrombotic diseases in none " $\mathrm{O}$ ' blood group individuals and " $\mathrm{O}$ " individuals are known to have a higher risk for inherited bleeding tendencies [21]. Thus, the suggested low thrombotic activity of blood group type $\mathrm{O}$, enhanced with the action of Apyrase and Tachykinin (antiplatelet factor and vasodilator respectively) secreted by mosquito, might create an easy blood flow into the mosquito gut. Rapid entry of the blood flow may accelerate a quick release of kairamone that leads to early aggregation of mosquitoes to blood source resulting a higher number of blood feeding.

Blood feeding behaviour of female mosquitoes are directly related with the reproductive activities. Gonzales et al. [15] have shown that serum proteins are the significant blood component that link to the egg production. After the entry of blood meal to the digestive tract, blood serum proteins are digested into amino acids. Amino acid, isoleucine is considered as the main player in the vitellogenesis process of mosquitoes. Lastly, these amino acids are subjected to absorb by fat bodies and they are converted into yolk precursor proteins [15]. Higher levels of yolk proteins give rise to higher number of eggs. Although the nature of host preference varies among the heamatophagous mosquitoes, the isoleucine low human blood preference of Ae. aegypti mosquito is scientifically proven [17]. However, although the quality of the blood source is generally believed to be contributed to reproductive activities, the study revealed no significant effect of reproduction in terms of egg production with respect to blood group types.

The differences among the blood groups are related with variations in oligosaccharides in structures present on erythrocyte membrane. Antigen A has an additional $\mathrm{N}$ acetylgalactosamine terminal which is absent in other two antigens. Antigen B is composed with an excess glycan molecule compare to antigen $\mathrm{O}$ and $\mathrm{A}$. Although there are differences in terms of oligosaccharides in erythrocyte membranes, the plasma composition differences among the blood groups is under explained. Few studies have been carried out to explain the role of carbohydrate in egg production. However there is no reproductive success other than the long lifespan in Ae. aegypti mosquitoes [24]. Thereby, the study reasons that the selection of blood group $\mathrm{O}$ may have other benefits such as longevity, survivorship and fitness other than egg production in Ae. aegypti female reproduction.

Finally the ultimate outcome of this study might open a new dimension to understand the behavioural patterns of $A e$. aegypti and provides more information to develop well managed effective vector control programs. Identification of mechanism behind the preference of Ae. aegypti on blood group $\mathrm{O}$ leads to the implementation of a new adult mosquito attractant trap that can be beneficial against to the increasing insecticide resistance. Since the findings that show the possibility of higher vector bites of blood group $\mathrm{O}$ individuals have a higher risk of developing vector borne diseases transmit by the Ae. aegypti. This might be a concern about personal protecting measures effectively to prevent mosquito bites. In addition if the relationship between preferred blood group and alteration of physiology or behaviour of mosquito is identified, national vector control programs could manipulate to control the vector population more effectively to control the current severe burden of dengue in Sri Lanka. Furthermore, the relationship of the dengue incidences and the distribution of the ABO brood groups in human population are essential to extrapolate the relationship of symptomatic and asymptomatic dengue infections to the individual blood groups.

\section{Conclusion}

The highest preference blood type of dengue vector mosquito Ae. aegypti is $\mathrm{O}$ according to the current analysis. However, most importantly the fecundity of these mosquitoes is independent with the blood type.

\section{Acknowledgements}

Authors are acknowledged to the Centre for Biotechnology, University of Sri Jayewardenepura for the laboratory and funding assistance to carry out the research.

\section{References}

[1] Soumahoro M. K., Fontenille D., Turbelin C., Pelat C., Boyd A., Flahault A. (2010) Imported chikungunya virus infection. Emerging Infectious Diseases 16: 162-163.

[2] Epidemiology unit. 2017. Dengue fever - Sri Lanka disease outbreak news [Internet]. Ministry of Health, Sri Lanka [accessed January 23, 2018]. Available from: http://www.who.int/csr/don/19-july-2017-dengue-sri-lanka/en/

[3] Scott T. W., Clark G. G., Amerasinghe P. H., Lorenz L. H., Reiter P., Edman J. D. (1993) Detection of multiple blood feeding patterns in Aedes aegypti (Diptera: Culicidae) during a single gonotrophic cycle using a histological technique. Journal of Medical Entomology 30:94-99.

[4] Burkot T. R., Graves P. M., Paru R., Lagog M. (1988) Mixed blood feeding by the malaria vectors in the Anopheles punctuates complex (Diptera: Culicidae). Journal of Medical Entomology 25: 205-213. 
[5] Chow E., Wirtz R. A., Scott T. W. (1993) Identification of blood meals in Aedes aegypti by antibody sandwich enzymelinked immunosorbent assay. Journal of American Mosquito Control Association 9: 196-205.

[6] Ngo K. A., Kramer L. D. (2003) Identification of mosquito blood meals using polymerase chain reaction (PCR) with order specific primers. Journal of Medical Entomology 40: 215-222.

[7] Kent R. J., Norris D. E. (2005) Identification of mammalian blood meals in mosquitoes by a multiplexed polymerase chain reaction targeting cytochrome B. American Journal of Tropical Medicine Hygiene 73: 336-342.

[8] Ponlawat A., Harrington L. C. (2005) Blood feeding patterns of Aedes aegypti and Aedes albopictus in Thailand. Journal of Medical Entomology 42: 844-849.

[9] Siriyasatien P., Pengsakul T., Kittichai V., Phumee A., Kaewsaitiam S., Thavara U., Tawatsin A., Asavadachanukorn P., Mulla M. S. (2010) Identification of blood meal of field caught Aedes aegypti (L.) by multiplex PCR. Southeast Asian Journal of Tropical Medicine and Public Health 41: 43-47.

[10] Anjomruz M., Oshaghi M. A., Sedaghat M. M., Pourfatollah A. A., Raeisi A., Vatandoost H., Mohtarami F., Yeryan M., Bakhshi H., Nikpoor F. (2014) ABO blood groups of residents and the $\mathrm{ABO}$ host choice of malaria vectors in southern Iran. Experimental Parasitology 136: 63-67.

[11] Shirai Y., Funada H., Takizawa H., Seki T., Morohashi M., Kamimura K. (2004) Landing preference of Aedes albopictus (Diptera: Culicidae) on human skin among ABO blood groups, secretors or nonsecretors, and $\mathrm{ABH}$ antigens. Journal of Medical Entomology 41: 796-799.

[12] Anand T., Kumar R., Saini V., Meena G. S., Ingle G. K. (2014) Knowledge and use of personal protective measures against mosquito borne diseases in a resettlement colony of Delhi. Annals of Medical and Health Science Research 4: 227-232.

[13] Duong V., Lambrechts L., Paul R. E., Ly S., Lay R. S., Long K. C., Huy R., Tarantola A., Scott T. W., Sakuntabhai A., Buchy P. (2015) Asymptomatic humans transmit dengue virus to mosquitoes. PNAS 112: 14688-14693.

[14] Richards S. L., Lord C. C., Pesko K. A., Tabachnick W. J. (2009) Environmental and biological factors influencing Culex pipiens quinquefasciatus Say (Diptera: Culicidae) vector competence for St. Louis encephalitis virus. American Journal of Tropical Medicine and Hygiene 81: 264-272.

[15] Gonzales K. K., Tsujimoto H., Hansen I. A. (2015) Blood serum and BSA, but neither red blood cells nor haemoglobin can support vitellogenesis and egg production in the dengue vector Aedes aegypti. Peer J 3: e938.

[16] Olayerni C. I., Ande T., Danlami G., Abdullahi U. (2011) Influence of blood meal type on reproductive performance of the malaria vector, Anopheles gambiae ss (Diptera: Culicidae). Journal of Entomology 8: 459-467.

[17] Naksathit A. T., Scott T. W. (1998). Effect of female size on fecundity and survivorship of Aedes aegypti fed only human blood versus human blood plus sugar. Journal of American Mosquito Control Association 14: 148-152.

[18] Lee S. H., Park G., Yang Y. G., Lee S. G., Kim S. W. (2009) Rapid ABO genotyping using whole blood without DNA purification. Korean Journal of. Laboratory Medicine 29: 231237.

[19] Wood C. S. (1976) ABO blood groups related to selection of human hosts by yellow fever vector. Human Biology 48: 337341 .

[20] Thiemann T. C., Wheeler S. S., Barker C. M., Reisen W. K. (2011). Mosquito host selection varies seasonally with host availability and mosquito density. PLOS Neglected Tropical Diseases 5: e1452.

[21] Jenkins P. V., O'Donnell J. S. (2006) ABO blood group determines plasma von Willebrand factor levels: a biologic function after all?. Transfusion 46: 1836- 1844.

[22] Lenting P. J., Pegon J. N., Christophe O. D., Denis C. V. (2010) Factor VIII and von Willebrand factor-too sweet for their own good. Haemophilia 16: 194-199.

[23] Gallinaro L., Cattini M. G., Sztukowska M., Padrini R., Sartorello F., Pontara E., Bertomoro A., Daidone V., Pagnan A., Casonato A. (2008) A shorter von Willebrand factor survival in $\mathrm{O}$ blood group subjects explains how $\mathrm{ABO}$ determinants influence plasma von Willebrand factor. Blood 111: 3540-3545.

[24] Day J. F., Edman J. D., Scott T. W. (1994) Reproductive fitness and survivorship of Aedes aegypti (Diptera: Culicidae) maintained on blood, with field observations from Thailand. Journal of Medical Entomology 31: 611-617. 